\title{
Leaching and structural modifications of Cr-bearing glasses
}

MARIONA TARRAGÓ, CHARLES LE LOSQ, PROF. ERIC D VAN HULLEBUSCH, PHD AND DANIEL NEUVILLE

Université de Paris, Institut de physique du globe de Paris, CNRS

Presenting Author: tarrago@ipgp.fr

Glass is traditionally considered a durable material (geologic glasses can be millions of years old) and is usually used as a container for liquids in households, industry and laboratories. Chromium is often used as a pigment in household glassware such as drinking glasses or dishes. Chromium gives glass a deep green color linked to the presence of $\mathrm{Cr}^{3+}$, which is expected to be the main redox species in the glass after production in an oxidizing furnace. $\mathrm{Cr}^{3+}$ is harmless to humans and it is implied (in trace amounts) in the metabolism of glucose. However, $\mathrm{Cr}^{6+}$ is a well-known carcinogenic, mutagenic and reprotoxic element, and it is not yet known if the alteration process can lead to redox changes. This issue is fundamental to glassmakers because European regulations (REACH, Food Contact Materials) increasingly require the industry to account for the safety of any element included in their products.

The alteration of glass has often been studied in terms of leached elements and subsequent analysis of the obtained liquids. However, the evolution of the glass structure at the molecular level during alteration is not yet well understood. The present study combines both approaches to analyze the alteration of a set of industrial glasses doped with $\mathrm{Cr}$ (between some ppm and 1 $\mathrm{wt} \%$ ) in an acidic aqueous medium of initial $\mathrm{pH} 2.3$ at 22 and 70 ${ }^{\circ} \mathrm{C}$ (a typical temperature of warm meals) for about 6 months. Glass dissolution causes an increase of $\mathrm{pH}$, leaching of the glass components into the liquid media (analyzed by ICP-MS) and surficial structural modifications that can be tracked by Raman spectroscopy. The main changes are due to the interaction of $\mathrm{H}_{3} \mathrm{O}^{+}$and $\mathrm{OH}^{-}$with the silicate network. Alteration changes the speciation of silicate $\mathrm{Q}^{\mathrm{n}}$-groups, the polymerization of the network and the bonds to the network modifiers. 